Elsevier Editorial System(tm) for Nuclear Inst. and Methods in Physics Research, A Manuscript Draft

Manuscript Number: NIMA-D-09-00829R1

Title: Time walk correction of CdTe detectors using depth sensing technique

Article Type: Research Paper

Section/Category: Data Acquisition and Control

Keywords: Time walk, Semiconductor detectors, Depth sensing

Corresponding Author: Research Associate MOHAMMAD NAKHOSTIN, PhD

Corresponding Author's Institution: University of Surrey

First Author: MOHAMMAD NAKHOSTIN, PhD

Order of Authors: MOHAMMAD NAKHOSTIN, PhD; P. M Walker, PhD; P. J Sellin, PhD

Abstract: A digital timing method aiming to minimize the time walk caused by the depth-dependent pulse shape variations in CdTe detectors has been developed. Detector pulses are digitized at the preamplifier stage and a full digital process is carried out to deduce and correct the time walk according to the interaction depth. A time resolution of $6.52 \mathrm{~ns}$ FWHM at an energy threshold of 150 $\mathrm{keV}$ with a CdTe detector $(10 \times 10 \times 1 \mathrm{~mm} 3)$ is achieved, which is close to the intrinsic resolution of the detector. The method improves the time resolution with no loss of detection efficiency and it is easy to implement. It is confirmed that the slow mobility and the short lifetime of the holes are major obstacles for further improvement in the timing performance of the CdTe detectors. The method is applicable to any semiconductor detector. 


\title{
Time walk correction of CdTe detectors using depth sensing technique
}

2

\author{
M. Nakhostin", P.M. Walker and P. J. Sellin \\ Department of Physics, University of Surrey, Guildford GU2 7XH, UK
}

\begin{abstract}
.
A digital timing method aiming to minimize the time walk caused by the depthdependent pulse shape variations in CdTe detectors has been developed. Detector pulses are digitized at the preamplifier stage and a full digital process is carried out to deduce and correct the time walk according to the interaction depth. A time resolution of $6.52 \mathrm{~ns}$ FWHM at an energy threshold of $150 \mathrm{keV}$ with a CdTe detector $\left(10 \times 10 \times 1 \mathrm{~mm}^{3}\right)$ is achieved, which is close to the intrinsic resolution of the detector. The method improves the time resolution with no loss of detection efficiency and it is easy to implement. It is confirmed that the slow mobility and the short lifetime of the holes are major obstacles for further improvement in the timing performance of the CdTe detectors. The method is applicable to any semiconductor detector.
\end{abstract}

17

Keywords: Time walk, Semiconductor detectors, Depth sensing

*Corresponding Author:

1 E-mail address: M.nakhostin@ surrey.ac.uk (M. Nakhostin),

Tel.:+44 1483686113

Fax: +441483686781 


\section{Introduction}

CdTe detectors have many applications in the fields of nuclear physics, medical imaging and industry. Advantages of these detectors include good energy resolution, good detection efficiency, room temperature operation and high detector spatial resolution using pixelated detector arrays comprised of very small elements. However, the timing performance of these detectors is not satisfactory, when compared to other detectors such as modern scintillation detectors. A good timing resolution is desired for various applications such as timing measurements in nuclear physics experiments and particularly for medical imaging using positron emission tomography (PET), where the CdTe detectors have shown to be a very promising candidate [1-3]. The timing performance of CdTe detectors is mainly limited by the variations in the shape of detector pulses and also electronic noise. Variation in the shape of detector pulses is due to the fact that the drift velocity of holes is approximately a tenth of that of electrons [4], and hence the shape of signals from a CdTe detector strongly depends on the interaction depth, i.e. where the electrons and holes are created in the detector. Variation in the shape of pulses leads to a significant timing error when the conventional timing methods such as leading edge or constant fraction discrimination (CFD) are used. This timing error is commonly referred to as "time walk".

The Amplitude and Rise-time Compensation (ARC) mode of operation is the most common method for minimizing the time walk caused by charge collection time variations $[5,6]$. ARC timing generates a timing marker that is independent of amplitude and rise-time, provided each pulse has a constant slope throughout its leading edge. However, real pulses from planar CdTe detectors exhibit constant slope only for the pulses with either minimum or maximum rise-time and many pulses are a mixture of these two shapes depending on the interaction depth. As a result of this deviation from the ideal linear rise, ARC timing does not provide perfect compensation for the rise-time variations of CdTe detectors.

In order to more completely remove the slope-dependent time walk, timing methods have been developed which operate based on an event-by-event correction of the time walk, according to the detector pulse shape. However, the practical use of these methods is limited by the complicated electronic system required or limitation for application to 
on-line measurements [7-11]. In a recent paper, it was shown that methods based on

2 digital processing of the detector signals can be used for improving the timing 3 performance of CdTe detectors [12]. In the present paper, a more detailed procedure for 4 digital correction of time walk caused by the variations in the shape of detector pulses is 5 presented. The method is based on the fact that the time walk is correlated with the risetime of pulses and, therefore, one can use a rise-time measurement to deduce the time walk, in order to improve the timing performance. This is analogous to the improvement of energy resolution of CdTe detectors with the depth of interaction correction $[13,14]$, because pulse rise-time reflects the depth of interaction, as well. The paper is organized as follows: In Section 2 the details of the method are described. Section 3 describes the experimental setup. Section 4 discusses the results and Section 5 summarizes the paper.

\section{Description of the method}

Due to the considerable difference in the mobility of electrons and holes, the output waveform of a preamplifier, connected to a CdTe detector, strongly depends on the interaction depth, where the charge is released in the detector crystal. The relation between the pulse rise-time and interaction depth is illustrated in Fig. 1. In addition to the pulse shape variations due to the different depths of interaction, further variation in the shape of detector pulses may be caused by the charge trapping and de-trapping in the detector crystal and also non-uniformities of the electric field [15]. As a result of the great variations in the shape of detector pulses, a significant time walk results, when the conventional CFD method is used. In the CFD triggering, the input pulse is inverted, delayed and then added to the attenuated original pulse to form a bipolar pulse with a zero crossing point. The discriminator detects this point and generates the corresponding timing output pulse. Since the time of zero crossing is a function of pulse rise-time, the time walk increases with the depth of interaction, which is defined as the distance of the interaction point from the detector cathode. The ARC timing tries to minimize this effect by applying the CFD technique to the early part of the pulse $[5,6]$. In this method, the detector pulses are shaped with a small shaping time constant, in order to reduce the noise and pulse duration, and the arrival time of the pulses is derived by setting a delay on the CFD, which is substantially shorter than the minimum rise-time of the pulses. The choice 
1 of short delay makes the zero-crossing occur in the early part of the pulse, leading to a 2 degree of immunity to the slope changes of pulses. However, the choice of delay is 3 practically limited because if the delay is very small, for some events the CFD process 4 can not transform the pulse to a biopolar pulse and the pickoff time would be generated 5 by the random noise on the pulse baseline. This type of timing error occurs for pulses with long rise-times and for pulses that exceed the threshold level by only a small amount. Consequently, the ARC timing can not completely compensate for the time walk of the pulses that experience a slope change early in their rise. This slope-changedependent time walk is a function of pulse shape, which is determined by the depth of interaction. Our correction procedure is to find this function and correct the time pickoff, generated by the ARC timing method. To do so, we group the pulses of similar rise-time together and the contribution of each group of pulses in the ARC timing of the CdTe detector is determined. This is done by employing a digital rise-time discriminator to group the pulses and a digital zero-crossing CFD for implementation of ARC timing. The details of the rise-time discrimination and procedure of time pickoff are described in the Sections 2.1 and 2.2. When the time spectra of the different groups of pulses are compared, the time walk is manifested in the systematic time delays between the time spectra, from which one can generate a set of correction factors for compensating the time walks in the time pickoffs of different groups of pulses. The correction factor for each group of pulses is determined by calculating the time difference between the peak position of the relevant time spectrum and the peak position of the spectrum associated with the fast pulses originating from the vicinity of the cathode, for which the time walk is negligible. The time walk is then corrected by subtracting the correction factor from the time pickoffs, derived by the standard ARC timing.

In addition to the charge collection time variations, the timing performance of a CdTe detector is affected by the electronic noise. However, in practice the CFD parameters are chosen to overcome the charge collection time variations, rather than less significant electronic noise. Since in our method the pulses are divided into several groups, the variation in the rise-time of pulses within each group of pulses is reduced and, therefore, the timing parameters can be set to yield a better suppression of noise. Applying a variable pulse shaping and CFD delay process to the different groups of pulses leads to 
$1 \quad$ systematic time offsets between the time pickoffs. Nevertheless, the time offsets are fixed

2 and, therefore, all the pulses can be synchronized by compensating the time offsets. This

3 is done by applying a second correction factor to the one derived for the time walk

4 correction. In practice, both the correction factors are combined together to form a total

5 correction factor, which is simply determined by comparing the peak position of the time

6 spectra. The complete procedure of timing is illustrated in Fig. 2. The procedure can be

7 summarized in three steps: (1) the rise-time of each pulse is evaluated, (2) a time pickoff

8 procedure, tailored in accordance with the pulse rise-time, is implemented and (3) a

9 correction factor is applied to correct the time walk and synchronize the time pickoffs. It

10 should be mentioned that the correction factors need to be determined by offline analysis

11 of the pulses. Nevertheless, the method can be used for online measurements once the

12 correction factors are known. In addition to time walk correction, the rise-time

13 information can be used to improve the energy resolution by compensating the pulse

14 deficit due to charge trapping inside the detector [16].

\subsection{Rise-time discriminator}

17 To evaluate the rise-time of pulses, a straightforward approach is to directly measure it 18 at the preamplifier output. However, the high frequency noise at the preamplifier output 19 limits the accuracy of such a measurement. We evaluated the rise-time of pulses by 20 measuring their peaking time after shaping with a digital CR-RC shaping filter [16]. A 21 CR-RC filter drastically reduces the noise, while still information on the rise-time of 22 pulses is reflected in the peaking time of the filtered pulse. This approach is 23 computationally very fast and at the same time prepares the pulses for extraction of 24 energy information as well. The peaking time of the pulses is determined by calculating 25 the time required for a pulse to change from $10 \%$ to $100 \%$ of the pulse amplitude. The 26 shaping time constant of the filter is decided in accordance with the best energy 27 resolution of the detector.

\subsection{Time pickoff procedure}

In the ARC timing of semiconductor detectors, before sending a pulse to a CFD, additional shaping is required to minimize the noise and reduce the duration of pulses. In 
1 the analogue regime this task is performed by using a timing filter amplifier that performs

2 independent differentiation and integration on the preamplifier pulses. In our study, pulse 3 shaping is performed using a digital CR-RC filter. The digital domain provides a flexible 4 approach in performing the integration and differentiation functions in order to set the 5 optimum shaping time constants. The optimal shaping time constant of the filter is

decided by comparing the time resolutions obtained using different shaping time constants. After pulse shaping, the pulses are sent to a digital version of zero-crossing CFD $[12,17]$ with an optimized delay on the CFD, corresponding to the ARC mode of operation. The CFD delay is optimized by performing multiple analyses on the pulses.

\section{Experimental setup}

The procedure of time walk correction is examined by using an experimental setup including a Schottky CdTe detector, a fast liquid scintillator detector and a fast digital oscilloscope as waveform digitizer. The schematic diagram of our experimental setup is shown in Fig. 3. A Schottky CdTe detector is placed against a small fast liquid scintillator (NE213 scintillator with a cylindrical volume of 2" long by 2" diameter), coupled to a Photomultiplier Tube (PMT) of type R329. The size of the Schottky CdTe detector is $10 \times 10 \times 1 \mathrm{~mm}^{3}$ and it is fabricated by evaporating platinum and indium as electrodes onto the surface of a CdTe wafer [18]. The detector is encapsulated in an aluminum box with a BNC connector for connection to a fast charge-sensitive preamplifier (Amptek 250 [19]). The preamplifier has a rise-time of $5 \mathrm{~ns}$. The detector is connected to the preamplifier through a de-coupling capacitor. Tests were performed using a ${ }^{22} \mathrm{Na}$ point source. The source and detector are placed in a way that radiation enters from the detector side. This geometry helps to capture more coincident events when the oscilloscope is triggered on the CdTe detector signals. The CdTe detector is operated at a bias voltage of $300 \mathrm{~V}$. In this operating condition, the maximum transit times for electrons and holes are $\sim 350 \mathrm{~ns}$ and $\sim 3.2 \mu \mathrm{s}$, respectively. At this voltage, the detector is fully depleted and the motilities of electrons and holes are saturated so that no further improvement in the signal rise time is observed at higher voltages. The measurements were performed at room temperature. To avoid the polarization phenomenon that affects Schottky CdTe detectors [20], the detector bias voltage was reset every $10 \mathrm{~min}$. 
The preamplifier and the PMT outputs are simultaneously digitized by means of the Lecroy WavePro7000 digital storage oscilloscope at a sampling rate of $5 \mathrm{GS} / \mathrm{s}$ and 8 bit resolution. The oscilloscope is set to trigger on the CdTe detector signals. Around 50,000 pairs of pulses were stored on the hard disk drive of the oscilloscope of which a considerable number are due to the $511 \mathrm{keV}$ coincidence $\gamma$-rays. The digitized pulses are transferred to a personal computer for offline analysis. The offline analysis is performed using a program written in MATLAB ${ }^{\circledR}$ language.

\section{Results}

In order to calibrate the energy scale of the system, the energy information of pulses is extracted after a CR-RC shaping filter with $3 \mu$ s shaping time constant. The energy spectrum of ${ }^{22} \mathrm{Na}$ is shown in Fig. 4. Performing the energy calibration of the system, using the $511 \mathrm{keV}$ positron annihilation peak and the $1275 \mathrm{keV} \gamma$-ray of ${ }^{22} \mathrm{Na}$, the energy threshold of the system is set at $150 \mathrm{keV}$. Then, a digital ARC timing procedure is initially performed on the pulses of the CdTe detector. The output pulses of the CdTe detector were shaped by a fast digital CR-RC filter with $15 \mathrm{~ns}$ shaping time constant. The arrival times of the pulses were then determined by feeding the shaped pulses to a digital zero-crossing CFD with $23 \mathrm{~ns}$ CFD delay and 0.24 attenuation factor, according to the best time resolution. With regard to the NE213 detector, since the pulses do not have the undesirable characteristics of variation in the pulse shape, no shaping is done on the pulses, and the arrival time is determined by direct application of a simple digital constant-fraction discriminator [15] to the PMT signals. The spectrum of the time difference between the arrival times of pulses from the two detectors is shown in Fig. 5. The spectrum represents a time resolution of 10.1 ns FWHM. Due to the negligible contribution of the scintillator detector to the time spectrum ( 1 ns FWHM), the FWHM of the time spectrum is considered to be the time resolution of the CdTe detector. The spectrum has an asymmetric shape and suffers from a tail, which is attributed to the slow rise-time events. The tail is a major problem in timing measurements with $\mathrm{CdTe}$ detectors, as a tight time window can cause a drastic loss in detection efficiency.

In the next step, the depth sensing technique is employed to correct the time walk. The depth of interaction is evaluated using the time discriminator that measures the peaking 
1 time of the pulses after shaping with the digital CR-RC filter, used for the energy 2 measurement. The shaping time constant of the filter is $3 \mu \mathrm{s}$, corresponding to the best 3 energy resolution. A distribution of peaking times is shown in Fig. 6. The distribution is 4 not symmetric due to the off-center position of the source in front of the detector. The 5 calculated peaking times are grouped into 6 bins as shown in the Figure and the timing spectrum of pulses associated with each bin is obtained against the scintillator detector. At this stage, the same pulse shaping and CFD procedures with those of Fig. 5 are applied to all groups of pulses. The results of these measurements, effectively as a function of depth of interaction, are shown in Fig. 7. The best timing resolution of less than $4.26 \mathrm{~ns}$ FWHM is recorded at depth parameter 1, corresponding to the pulses from $\gamma$-rays interacting close to the cathode of the detector. It is seen that the time resolution decreases as the depth of interaction increases and the widest spectrum results from the group of pulses originating from the vicinity of the anode, with a time resolution of $\sim 50$ ns FWHM. The relative positions of the time spectra, associated with different depths of interactions, are shown in Fig. 8. Due to the walk in the time pickoffs, the peak positions show a marked dependency on the depth of interaction. In fact, a time difference of $20 \mathrm{~ns}$ is observed between the time spectra of pulses originating from the two ends of the detector. The depth effect is corrected by deducing a correction factor from the CFD outputs of each group of pulses. The correction factor of each group is the difference between the peak position of the associated time spectrum and the time spectrum of the pulses associated with the depth parameter 1 . The time spectrum resulting from the depth correction method is shown in Fig. 9. The time resolution improves to $7.12 \mathrm{~ns}$ and the tail of the spectrum caused by the slow events is considerably diminished. It is worth mentioning that the improvement is achieved without affecting the detection efficiency. If the detection efficiency is not so critical, the spectra with distorted timing resolution can be rejected to use only the best time resolution. This is a similar procedure to the slow Rise-time Reject (SR) timing mode, which is provided in the standard analogue CFD modules.

In addition to the time walk correction, a further improvement in the procedure of time pickoff is to minimize the noise effect. For this purpose, pulse shaping and CFD parameters are separately optimized for each group of pulses. The signal-to-noise ratio is 
1 improved by making a slight increase in the shaping time constant of the digital timing 2 filter, in accordance with the rise-time of the pulses. A considerable increase in the CFD 3 delay is also made to make the zero crossing at the optimum point of slope-to-noise ratio. $4 \quad$ Again the time spectra of different groups of pulses are obtained and the correction 5 factors for compensating the time walk and the time offset caused by the different 6 procedures of time pickoff are determined by comparing the new time spectra. The 7 overall time spectrum, after applying the final correction factors, is shown in Fig. 10. A 8 time resolution of $6.52 \mathrm{~ns}$ FWHM is achieved. Table 1 presents a comparison of time

If the noise cannot be reduced, the minimum timing error is determined by the maximum slope on the analog pulse. Due to the low mobility of holes, for the pulses that have their time pickoff determined by the hole's contribution to the pulse, the achievable 
1 slope-to-noise ratio is limited and therefore greater timing errors are expected. In fact, the 2 pulse slope for hole-dominant pulses is around ten times slower than the electron3 dominant pulses, which is in agreement with the timing results. The timing resolution of 4 pulses from depth parameter 6 is around ten times larger than that of the pulses from the 5 depth parameter 1. Further deterioration of timing performance stems from hole trapping. 6 Because the hole's lifetime is so much shorter than the hole transit time, the pulse 7 amplitude is very dependent upon the depth of interaction and the collected charge is 8 significantly reduced with increasing path length. This affects the time resolution by 9 changing the shape of pulses and reducing the slope-to-noise ratio. Fig. 11 shows the 10 energy spectra as a function of interaction depth. The peak positions show a marked 11 depth dependence which is due to the charge trapping effect. Since both rise-time and 12 pulse amplitude are correlated with the depth of interaction, in a similar manner with the 13 time walk correction, one can use the depth data to deduce the charge collection 14 efficiency and thus improve the spectroscopic performance.

\section{Summary}

\section{Acknowledgment}

The authors would like to thank the Cyclotron and Radioisotope Center in Tohoku University in Japan for providing the detectors and digitizer. We acknowledge the support from the UK STFC and AWE plc.

\section{References}

[1] G. S.Mitchell, S.Sinha, et.al., IEEE Trans. Nucl. Sci. NS-55 (2008) 870. 
1 [2] K.Ishii, et al., Nucl. Instr. and Meth. A 576 (2007) 435.

2 [3] Y. Ueno, et al., IEEE Trans. Nucl. Sci. NS-56 (2009) 24.

3 [4] Y. Okada, et al., IEEE Trans. Nucl. Sci. NS-49 (2002) 1986.

$4 \quad$ [5] R.L. Chase, Rev. Sci. Instrum. 39 (1968) 1318.

5 [6] Z.H. Cho and R. L. Chase, Nucl. Instr. and Meth. 98 (1972) 335.

6 [7] S.Y.Kim, et al., Nucl. Instr. and Meth. A 414 (1998) 372.

7 [8] L. Heilbronn, et al., Nucl. Instr. and Meth. A 522 (2004) 495.

8 [9] J. P. Fouan and J. P. Passerieux, Nucl. Instr. Meth. 62 (1968) 327.

9 [10] S. Bose, et al., Nucl. Instrum. Meth. A 295 (1990) 219.

$10 \quad$ [11] A. R. Frolov et al., Nucl. Instrum. and Meth. A 356 (1995) 447.

11 [12] M. Nakhostin, K. Ishii, et al., Nucl. Instr. and Meth. A 606 (2009) 681.

12 [13] Z. He, et al., Nucl. Instr. and Meth. A 380 (1996) 228.

13 [14] Z. He, R. Vigil, Nucl. Instr. and Meth. A 492 (2002) 387.

14 [15] C. Bargholtz, et al., Nucl. Instr. and Meth. A 471 (2001) 290.

15 [16] M. Nakhostin, et al., Nucl. Instr. and Meth. A 615 (2010) 242.

16 [17] M. A. Nelson, B. D. Rooney et al., Nucl. Instr. and Meth. A 505 (2003) 324.

17 [18] K. Matsumoto and T. Takahashi et al., IEEE Trans. Nucl. Sci. NS-31 (1998) 556.

18 [19] http://www.amptek.com/a250.html .

19 [20] H. Toyama and A. Higa et al., Jpn. J. Appl. Phys. 45 (11) (2006) 8842.

$20 \quad$ [21] K. G. Giboni, et al., Nucl. Instr. and Meth. A 450 (2000) 307.

\section{Tables}

23 Table 1. Results of time resolution measurements using $1 \mathrm{~mm}$ thick CdTe detectors

\section{Figures Captions}

Figure 1. Relationship of interaction depth to detector pulse shape. Three interaction 28 positions are shown leading to three different waveforms. Position 1 illustrates the case 29 where charge is produced very close to the cathode and the pulse is due to the motion of 30 the electrons. Position 2 is a case that charge is produced at a mid-point between the 31 anode and cathode so that the pulse is due to both electrons and holes. An abrupt slope 
1 change is observed as the electrons reach to their electrode. In case 3 , the charge is

2 produced very close to anode and the pulse is due entirely to the motion of the holes.

5 depth data can be used for improving the energy resolution through compensating for

6 charge trapping.

8 Figure 3. Schematic diagram of the setup used for the timing measurements.

10 Figure 4. The energy spectrum of ${ }^{22} \mathrm{Na}$ measured with the CdTe detector. The energy 11 threshold is approximately $150 \mathrm{keV}$.

13 Figure 5. Timing spectrum between the Schottky CdTe detector and the liquid 14 scintillator. The width of the spectrum is $10.1 \mathrm{~ns}$ FWHM. The energy threshold is $\sim 150$ $15 \mathrm{keV}$.

17 Figure 6. Distribution of signal peaking time. The asymmetric shape of the spectrum 18 stems from the off-center position of the source in front of the detector.

Figure 7. Timing spectrum vs depth of interaction. The time resolutions (FWHM) of the spectra from anode to cathode are, respectively, 4.26, 5.55, 8.45, 13.63, 25 and $50 \mathrm{~ns}$.

Figure 8 . The relative position of time spectra associated with different parts of the detector.

Figure 9. Comparison of timing spectra before and after time walk correction. (A) standard ARC timing, (B) after time walk correction. 
$1 \quad$ Figure 11. Energy spectra of ${ }^{22} \mathrm{Na}$ as a function of depth parameter.

2 

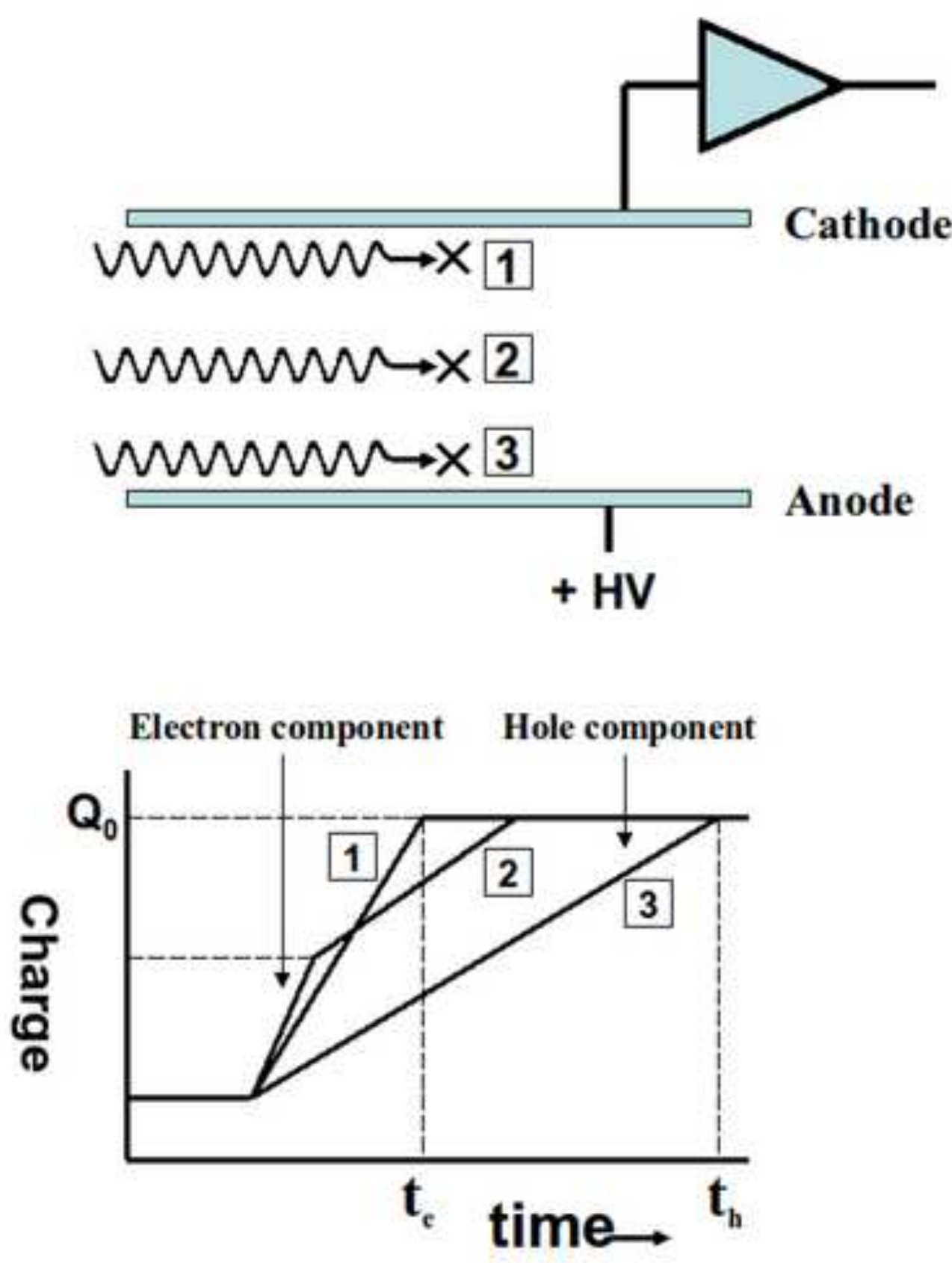


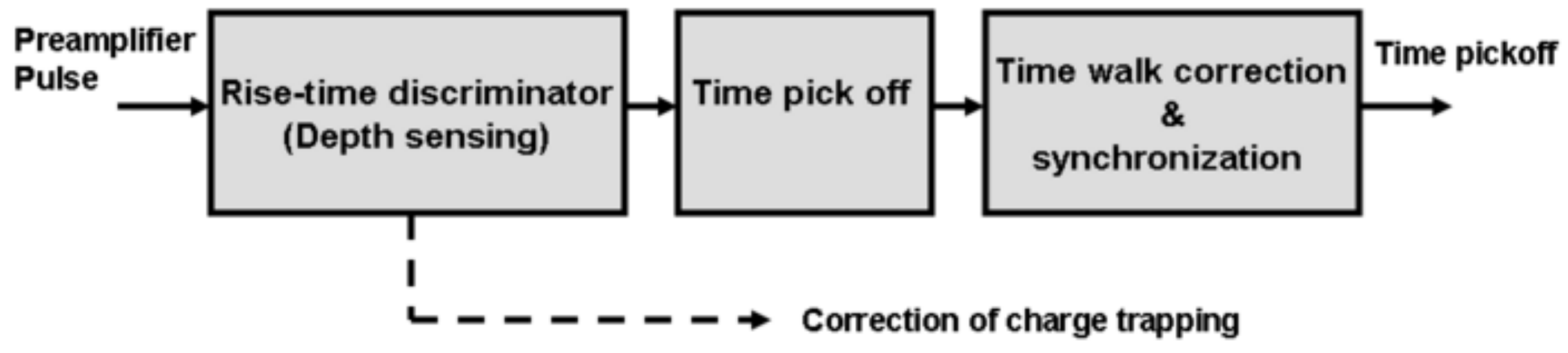




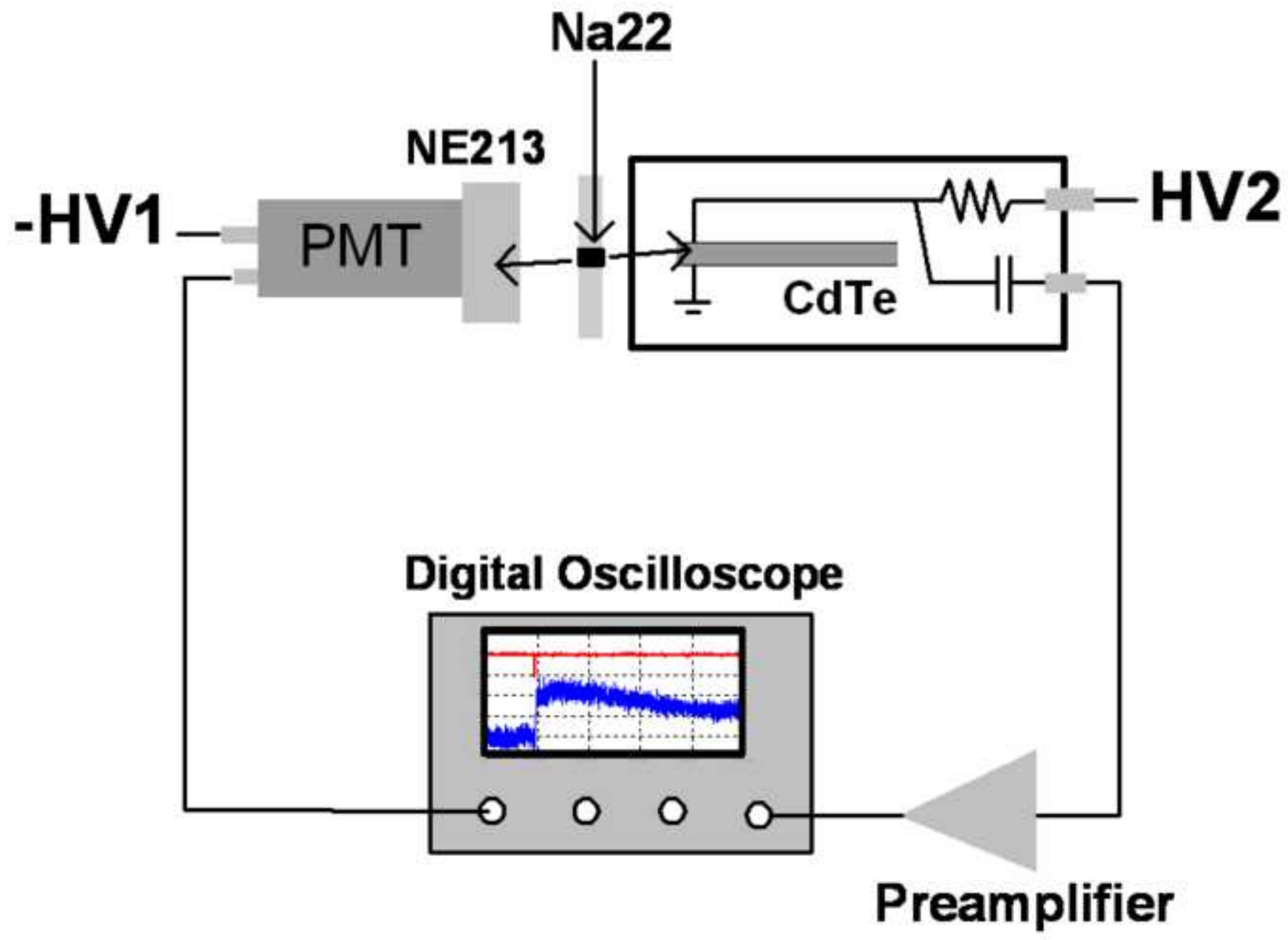




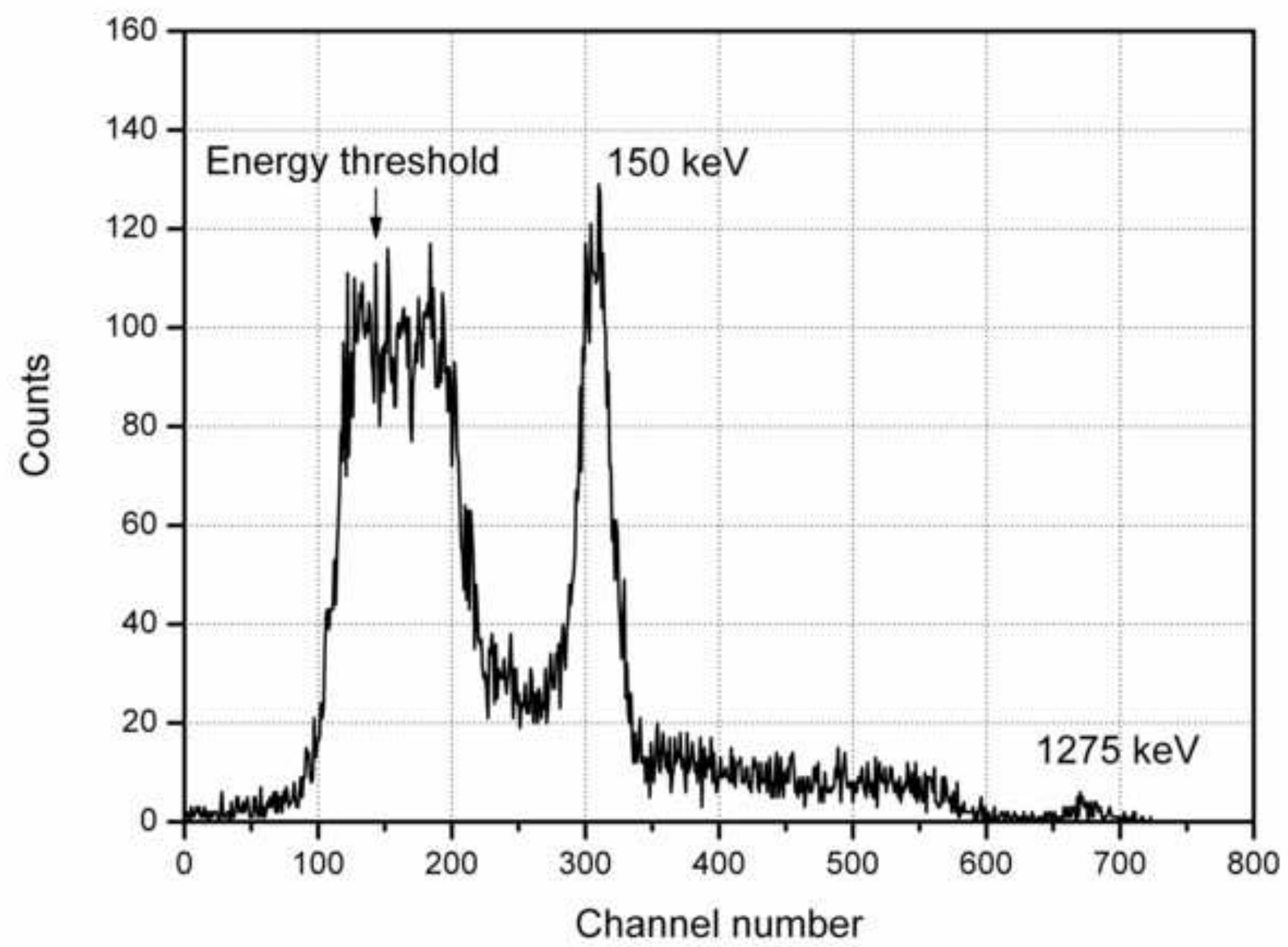




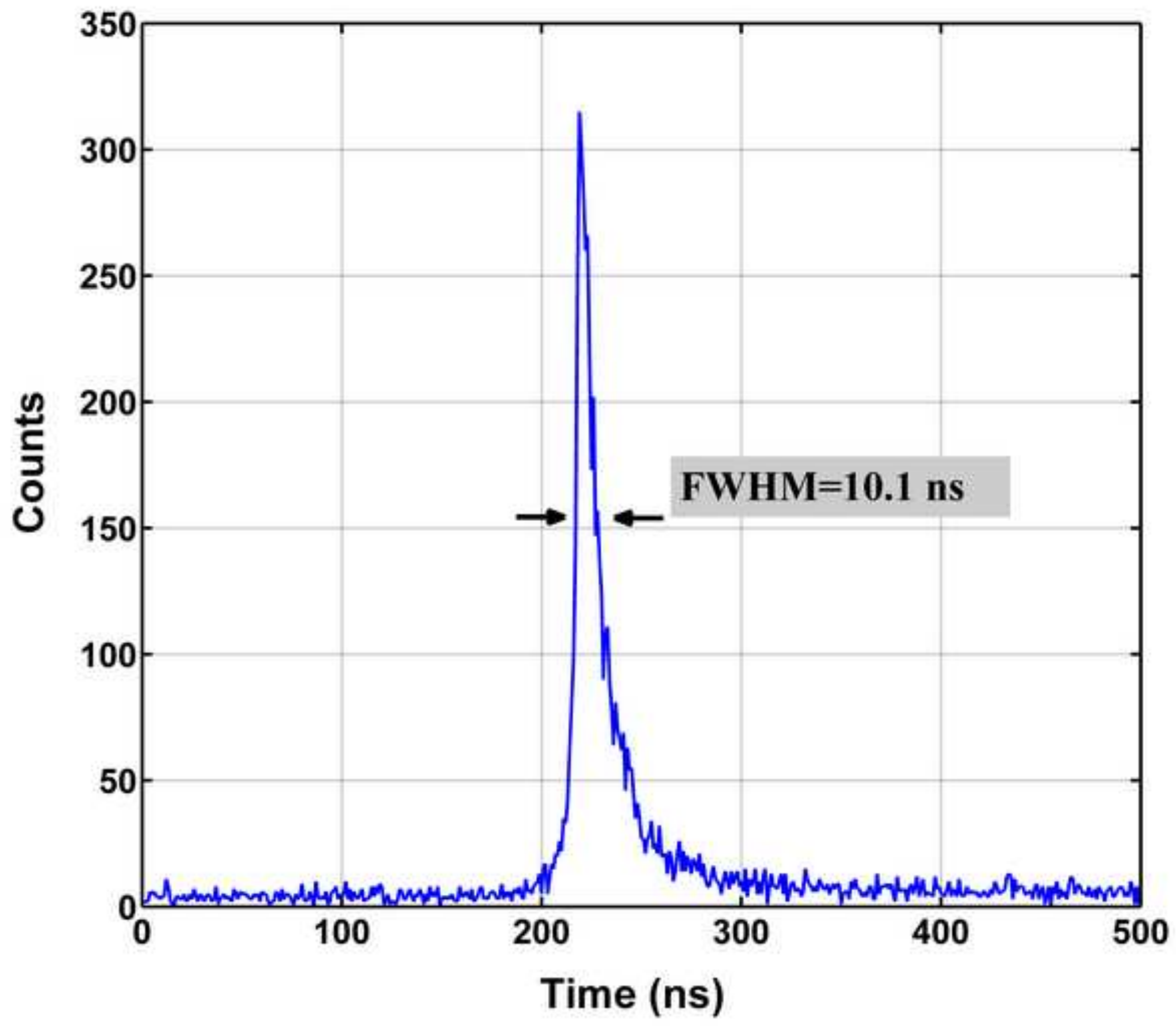


Click here to download high resolution image

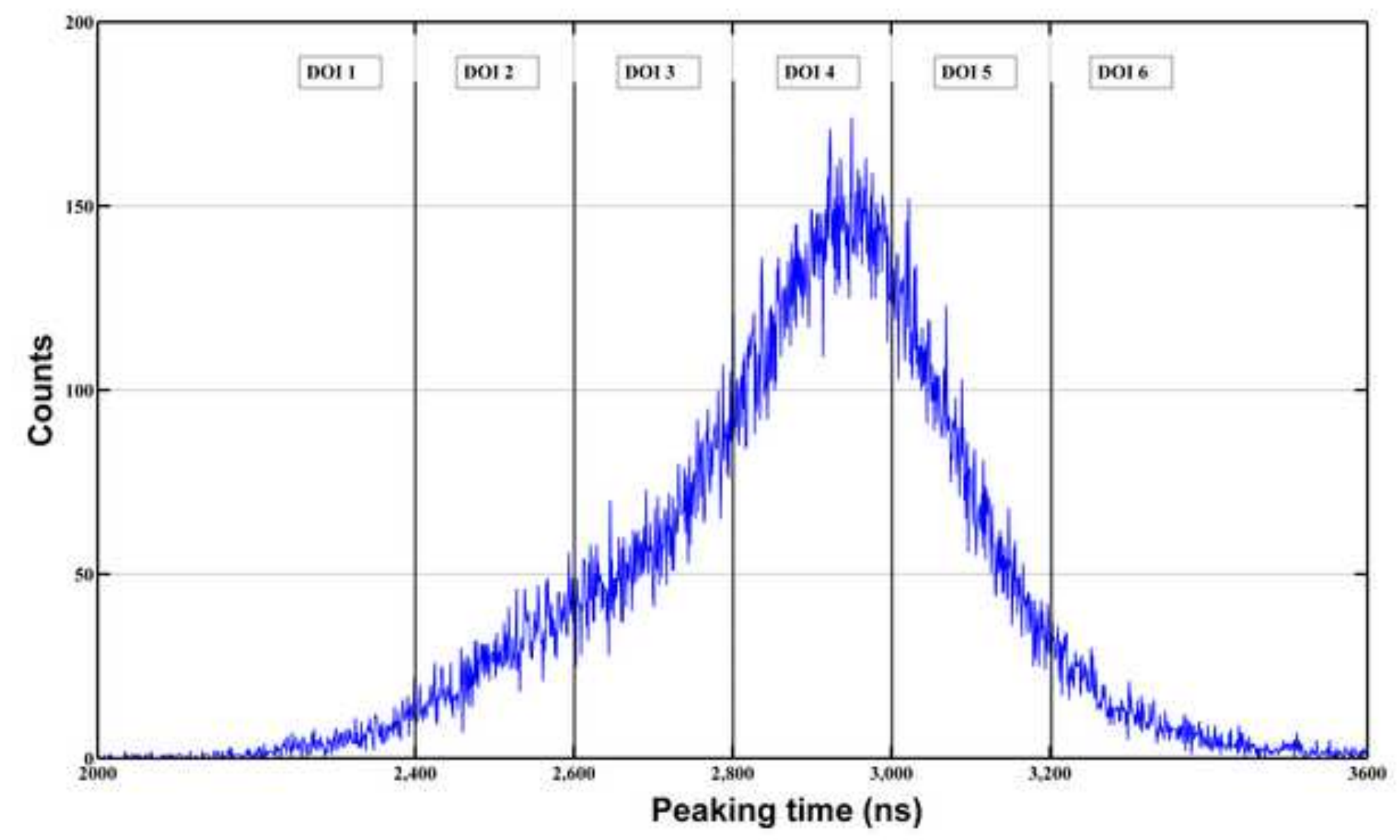


Click here to download high resolution image

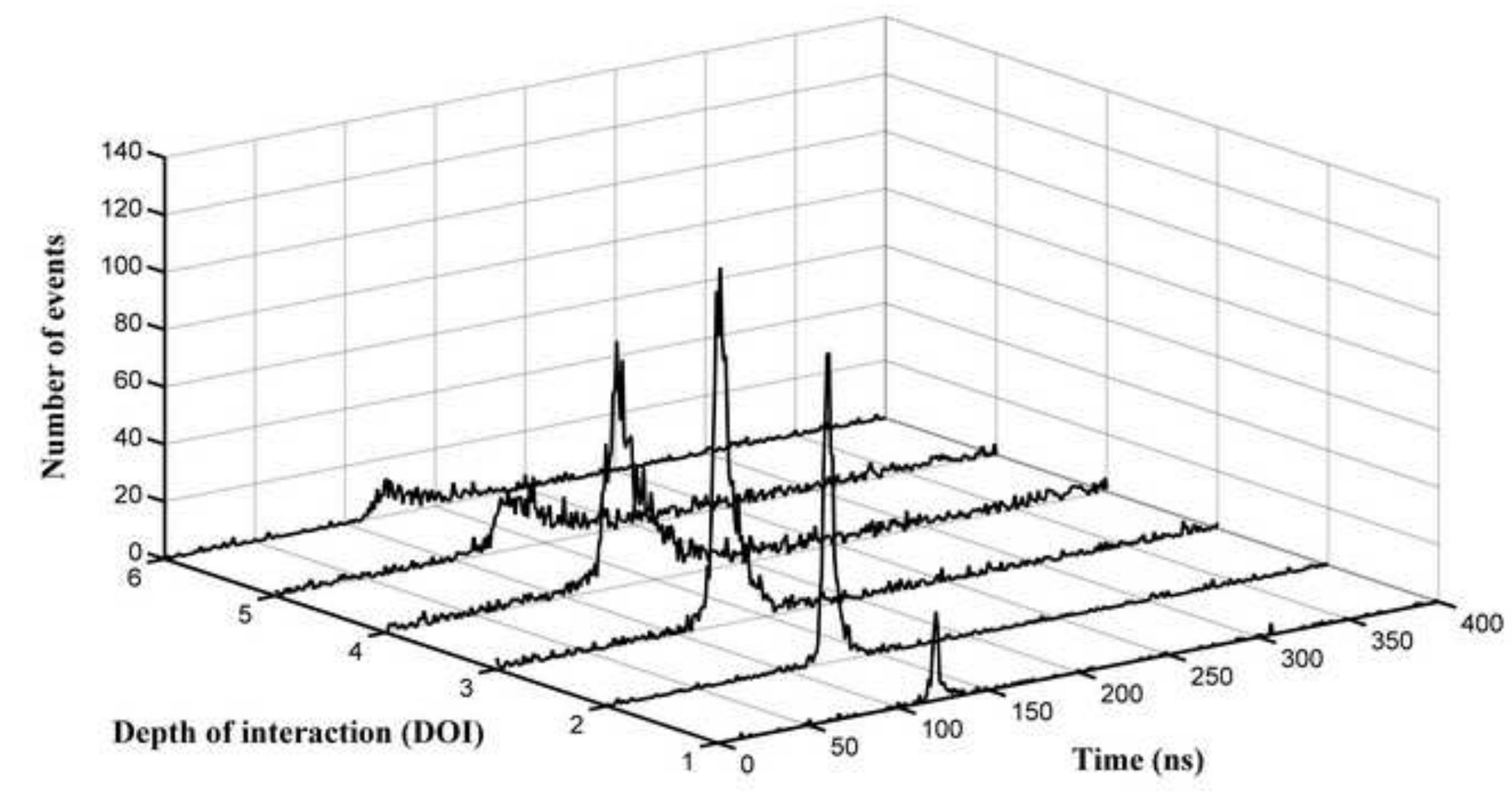




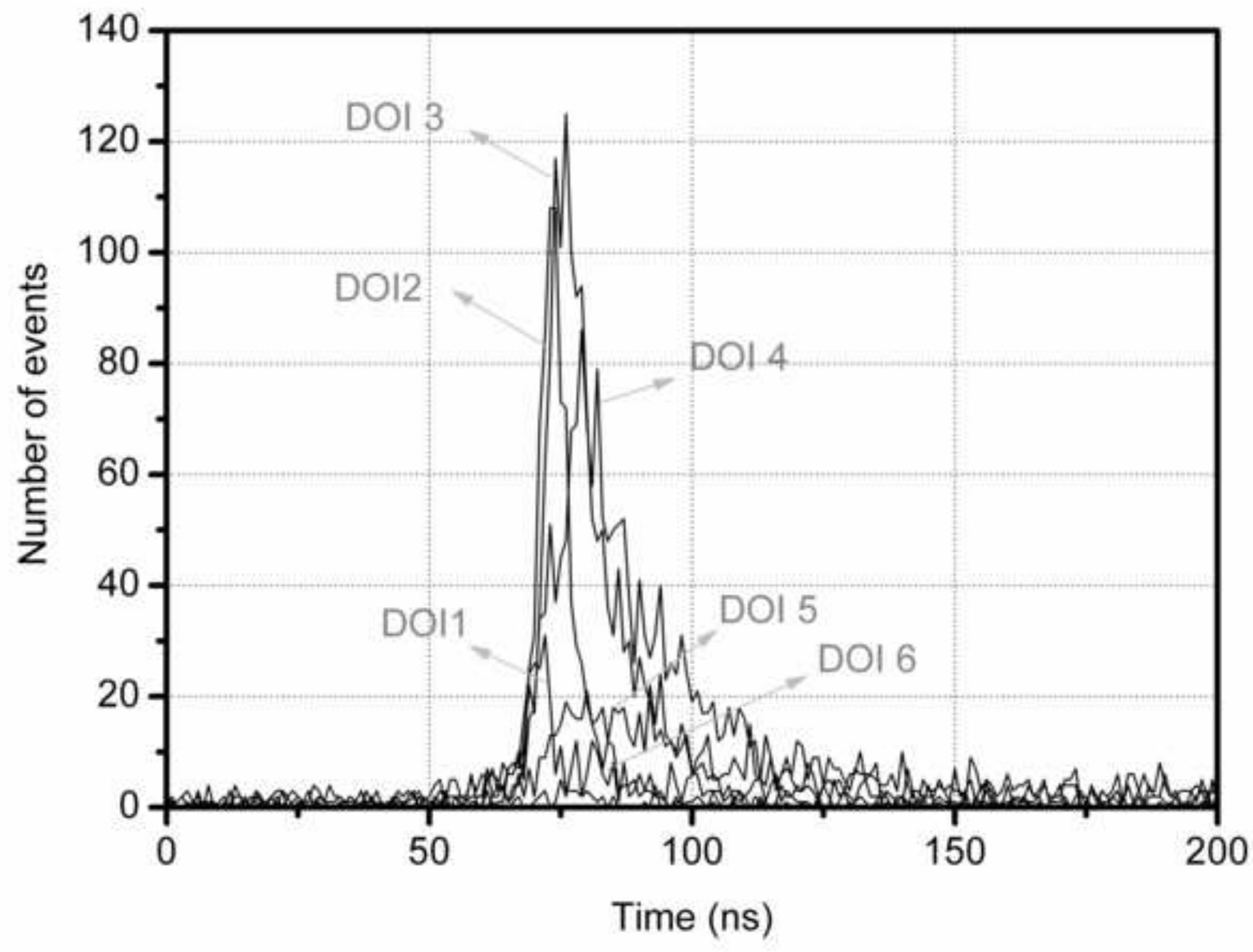



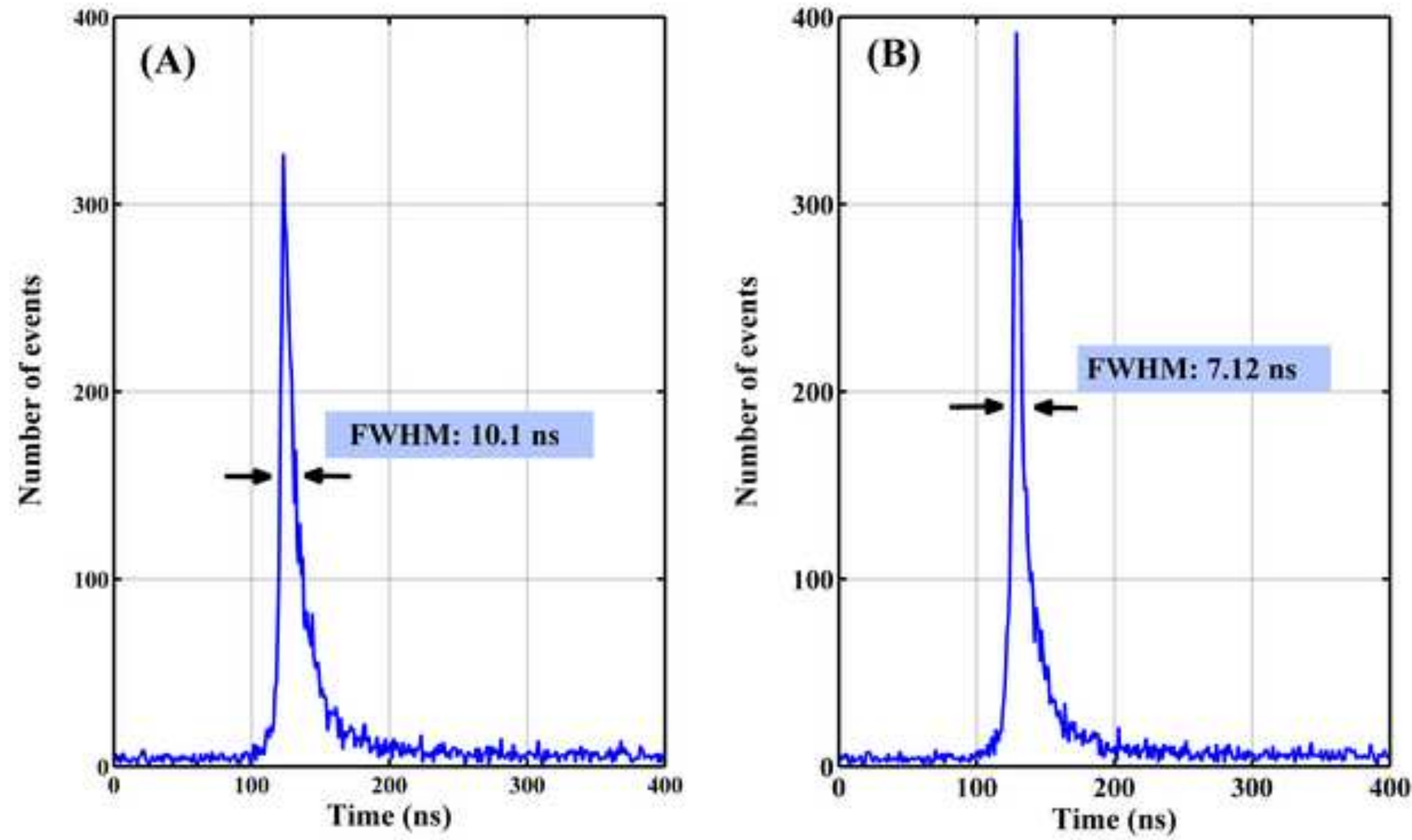

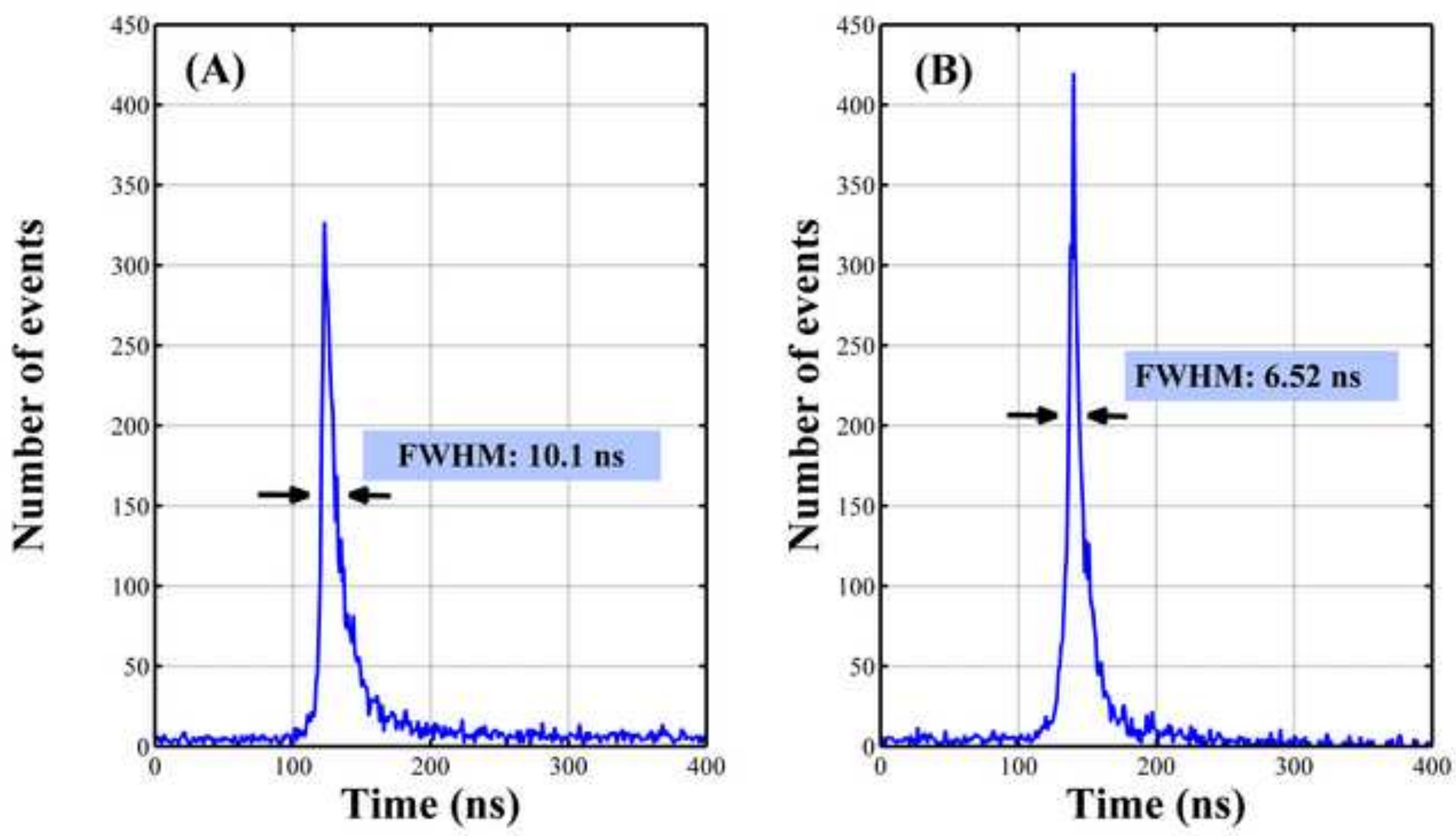


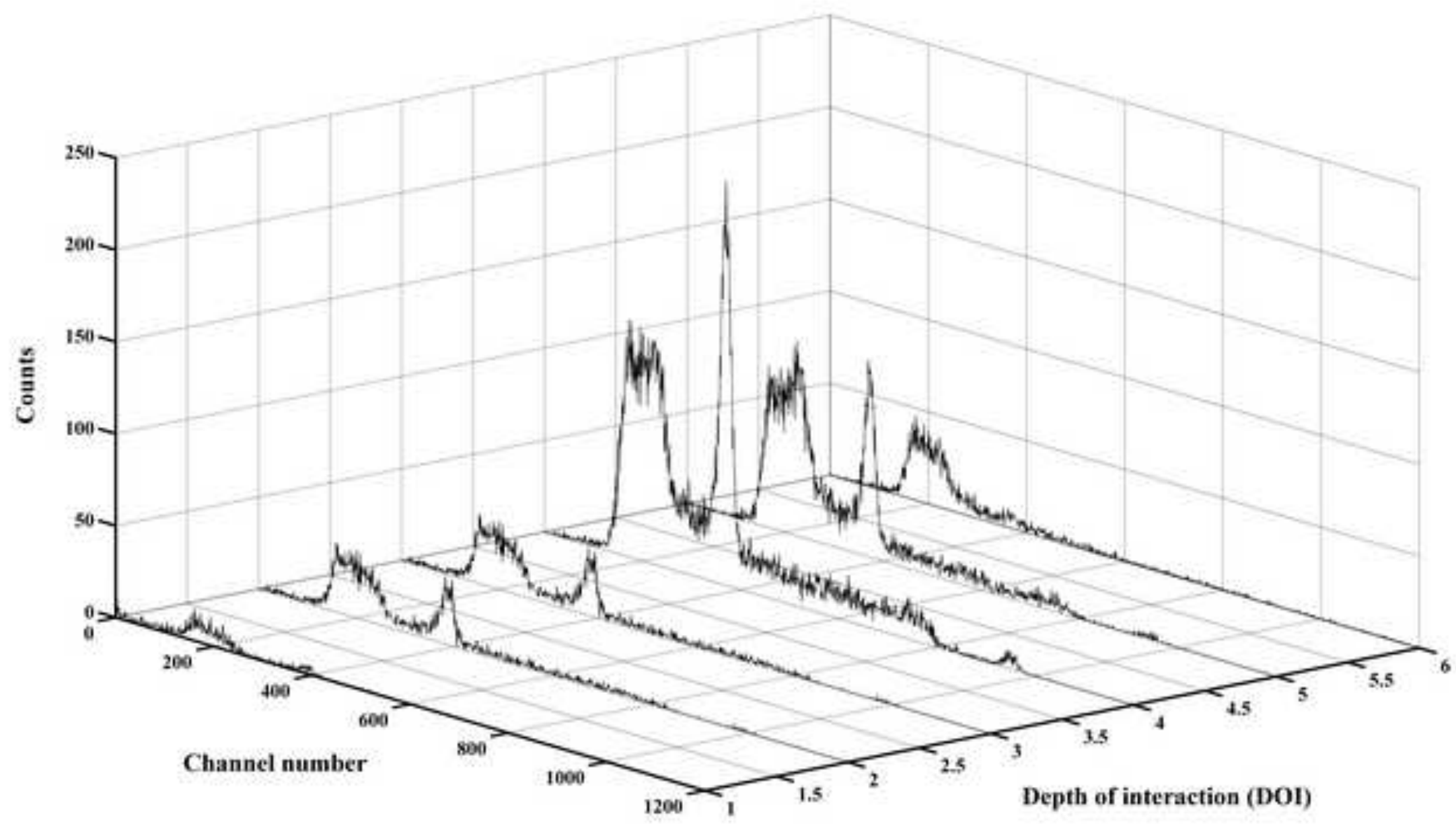




\begin{tabular}{|c|c|c|c|c|}
\hline Resolution & $\begin{array}{c}\text { Energy } \\
\text { threshold }\end{array}$ & Method & Temperature & Reference \\
\hline $6.52 \mathrm{~ns}$ & $150 \mathrm{keV}$ & Digital & $\begin{array}{c}\text { Room } \\
\text { Temp. }\end{array}$ & $\begin{array}{c}\text { Present } \\
\text { Work }\end{array}$ \\
\hline $4.255 \mathrm{~ns}$ & $450 \mathrm{keV}$ & Analog & $\begin{array}{c}\text { Room } \\
\text { Temp. }\end{array}$ & {$[3]$} \\
\hline $7.2 \mathrm{~ns}$ & $150 \mathrm{keV}$ & Digital & $\begin{array}{l}\text { Room } \\
\text { Temp. }\end{array}$ & {$[12]$} \\
\hline $9.6 \mathrm{~ns}$ & $150 \mathrm{keV}$ & Analog & $-20 \mathrm{C}$ & {$[21]$} \\
\hline
\end{tabular}


List of changes, reviewer \#2

1. In order to be able to compare the results of this work with the results of the previous paper, the energy threshold of the measurements was set at $150 \mathrm{keV}$, the same as that of a previous paper ( NIMA vol 606, p. 681, 2009). Results obtained under the new conditions were compared with the literature data for 1 $\mathrm{mm}$ thick CdTe detector. Corrections in page 3, lines 1-3 and page 9, lines 715.

2. Page 2 , line 10 , new reference was included. In discussion the result of the reference was added, page 9, line 7-15 and Table 1.

3. Page 2, line 13, a reference was added.

4. Page 6 , line 17, type of PMT was indicated. 
List of changes, reviewer \#1

Page 1, line 5, pixilated was corrected.

Page 4, line 16, Sections 3.1 and 3.2 was corrected to sections 2.1 and 2.2.

Page 5, line 16, corrected.

Page 5, line 29, corrected.

Page 6, lines 27-29, it was explained that at this voltage motilities are saturated therefore, an improvement of signal rise time can not be expected.

Page 8 , line 13, cathode was replaced by anode.

Page 8, line 3-5, a distribution of signal peaking time was included.

Page 8 , line 4-5, and page 66, lines 22-25, and figure 3 , corrections was made to clarify that the interactions do not occur uniformly across all depth due the geometry of the experiment.

Page 9 lines 14-15, explanation was included to clarify the intrinsic resolution. 